\section{$(x)$ \\ HORIZONTES}

www.revistahorizontes.org
Horizontes. Revista de Investigación en Ciencias de la Educación Https://doi.org/10.33996/revistahorizontes.v4i13.91

\title{
Diseño de material instruccional para la enseñanza de algoritmia y programación en las prácticas profesionales de profesores en formación
}

\author{
Design of instructional material for teaching algorithm and programming in \\ the professional practices of teachers in training
}

\author{
Esther Carpio \\ profa.esther@yahoo.es \\ ORCID ID: https://orcid.org/0000-0002-6684-325X
}

Universidad Pedagógica Experimental Libertador. Instituto Pedagógico de Caracas, Venezuela

Recibido octubre 2019 | Revisado nov - dic 2019 | Publicado 01 de enero 2020

\section{RESUMEN}

El estudio refiere el desarrollo de una solución instruccional que permita a los estudiantes de la Especialidad de Informática del IPC, cursantes de las Prácticas Profesionales, minimizar debilidades pedagógicas $\mathrm{y}$ especializadas en la enseñanza de asignaturas asociadas a la algoritmia y programación. La investigación realizada se caracteriza por ser un proyecto factible, dirigido a la realización de propuestas y modelos encausados hacia la solución de problemas y necesidades detectadas. Fue considerado el Modelo de Desarrollo Instruccional propuesto por Szczurek (1989), el cual comprende cuatro fases: Estudio de Necesidades (Modelo de Müller, 2003), Diseño de la Solución, Implementación de la Solución y Evaluación de cada una de las fases antes citadas. Para el diseño de la solución se empleó el Modelo de Dorrego (1994) para la realización del material instruccional y el Modelo de Aprendizaje a Través de la Práctica de Schank, Berman y Macpherson (citado por Reigeluth, 1999) para el diseño de la sesión de clase. La fase de Implantación no fue abordada en éste estudio, se propone el Modelo de Zabatta (1997) para su posterior desarrollo. Para la evaluación del sistema se utilizó el Modelo de Stufflebeam (1987, citado por Abdala, 2007).

Palabras clave: Material instruccional; prácticas profesionales; algoritmia y programación; enseñanza y aprendizaje

\section{ABSTRACT}

The study refers to the development of an instructional solution that allows students of the Computer Science Specialty of the IPC, students of Professional Practices, minimize pedagogical weaknesses and specialized in teaching subjects associated with the algorithm and programming. The research carried out is characterized as a feasible project, aimed at the realization of proposals and models aimed at solving problems and needs detected. The Instructional Development Model proposed by Szczurek (1989) was considered, which includes four phases: Needs Study (Müller Model, 2003), Solution Design, Solution Implementation and Evaluation of each of the phases mentioned above For the design of the solution the Dorrego Model (1994) was used for the realization of the instructional material and the Learning Model through the Practice of Schank, Berman and Macpherson (cited by Reigeluth, 1999) for the design of the session of class. The Implantation phase was not addressed in this study, the Zabatta Model (1997) is proposed for further development. For the evaluation of the system, the Stufflebeam Model (1987, cited by Abdala, 2007) was used.

Keys words: Instructional material, professional practices, algorithm and programming, teaching 


\section{INTRODUCCIÓN}

La incursión y auge de la informática en el quehacer humano es una realidad ineludible, ha impactado las bases de la sociedad y derivado en la necesidad de hacer cambios para formar al hombre, al ciudadano que responda al nuevo orden social donde las herramientas informáticas y el conocimiento enmarcan el camino de ésta y de las generaciones venideras. Éste contexto, colma de retos a los sistemas educativos del mundo considerando los nuevos paradigmas y formas de pensamiento que se desprenden del ámbito tecnológico y digitalizado.

Así pues, la informática como tema propio de enseñanza, como área de conocimiento en las instituciones educativas, demanda cada día más profesionales para cubrir las solicitudes de los centros educativos, que van desde el estudio de las redes de computadoras, las bases de datos, los sistemas informáticos, hasta la algoritmia y programación.

La educación informática va más allá del uso de recursos tecnológicos para facilitar actividades instruccionales y más que enseñar a utilizar herramientas tecnológicas para llevar a cabo una tarea: ésta aborda la enseñanza de la informática como ciencia, lo cual requiere de profesionales especializados y con preparación pedagógica para abordar la enseñanza de un área caracterizada por altos niveles de abstracción para su comprensión (Beccaria y Rey, s.f).

En particular, la algoritmia y programación es una de las áreas de la informática de mayor complejidad. En éste ámbito, los estudiantes han demostrado dificultades para su compresión por los niveles de abstracción requeridos y su fundamentación matemática (Oviedo, 2002). De lo anterior, son varios los factores que posicionan ésta área de la informática como difícil; por lo que es frecuente el abordaje de la misma con cierto rechazo.

En tal sentido, para la enseñanza de la algoritmia y programación no solo es requerida la puesta en práctica de los conocimientos especializados del docente, también de habilidades pedagógicas para facilitar el aprendizaje.

\section{Formación docente en la especialidad Informática}

Las demandas en la enseñanza de la informática han ido en aumento durante las últimas décadas, en respuesta a los cambios cotidianos generados por la incursión de herramientas tecnológicas en el aula de clases y en otros contextos asociados a la educación. Tal escenario ha repercutido directamente en la forma de ejercer la docencia y en la formación requerida para atender a las exigencias de la sociedad tecnificada del mundo actual.

Punceles (2008) hace referencia a la formación de profesionales en áreas técnicas y en particular a la preparación pedagógica para la enseñanza de alguna de éstas áreas de conocimiento. Concretamente señala:

...profesionales de diferentes áreas como contadores, administradores e informáticos que ejercen la docencia y que a su vez han realizado algún componente docente que les facilita de una u otra forma la práctica pedagógica...este cuerpo de profesores requiere de la formación continua para dar respuesta a las exigencias de la educación superior contemporánea y convertirse en agentes claves de transformaciones venideras (s.p). 
Así pues, aunque aborda la formación docente a nivel universitario permite identificar una realidad común en otros niveles del sistema educativo, donde los especialistas en áreas como la informática realizan estudios pedagógicos complementarios para ejercer profesionalmente la docencia. Además, se evidencia la ineludible actualización a la que deben responder quiénes enseñen informática y áreas afines, debido a la naturaleza cambiante de ésta ciencia.

Asimismo, la creciente demanda de educadores en informática ha generado la incorporación de profesionales en el área que no necesariamente poseen conocimientos pedagógicos para la enseñanza de la misma, atribuyéndose múltiples causas a la carencia de profesores en ésta especialidad y aumentando la necesidad de formación docente.

Aunado a esta realidad, Chiarani (2001) sostiene: "Es difícil encontrar docentes trabajando con la informática como medio didáctico, notándose muy poca o ninguna relación con los contenidos curriculares, porque la materia era dada por técnicos informáticos o ingenieros en su mayoría con escasos conocimientos pedagógicos".

En consecuencia, la introducción de la informática en los niveles del sistema educativo, ya sea como objeto de estudio, herramienta de trabajo o medio de enseñanza solicita la formación del personal docente capaz de enfrentar esta tarea (Pereira, 2005).

Adicionalmente, el autor señala la necesidad de considerar los factores sociales que determinan la urgente formación de docentes de informática; entre ellos:

- La introducción de la computación en todos los niveles de enseñanza. Esto trae consigo la necesidad de tener los maestros que sean capaces de formar el personal calificado para poder trabajar en cada uno de estos lugares.
- El éxodo de muchos de los profesores que ya tenían esa formación informática hacia otras esferas donde se introdujo la computación, en las cuales son necesarios también.

Por otro lado, la UPEL (2002) como una de las pocas casas de estudio que egresan docentes especializados en informática, establece que el egresado de ésta especialidad debe recibir formación para:

- Aplicar los conceptos de la informática en situaciones educativas.

- Aplicar metodologías, técnicas y procedimientos, para favorecer el proceso de enseñanza-aprendizaje de la Informática.

- Poseer una visión integral de la Informática y su proyección en el desarrollo social.

- Facilitar los procesos de autoevaluación y autoconocimiento que sustenta el proceso de enseñanza-aprendizaje.

- Diseñar estrategias para dirigir el proceso de enseñanza-aprendizaje de la Informática.

- Aplicar estrategias para facilitar el proceso de enseñanza-aprendizaje en el campo de la Informática.

- Analizar críticamente los problemas relacionados con el aprendizaje de la Informática en los diferentes niveles $\mathrm{y}$ modalidades del sistema educativo.

- Controlar la administración de los recursos computacionales a fin de obtener el máximo rendimiento de los mismos, desde el punto de vista académico, administrativo y social.

Por consiguiente, reconociendo la existencia de un perfil definido por las casas de estudios para formar docentes en informática como es el caso de la UPEL, se evidencia la necesidad de disminuir la brecha 
entre el Deber Ser antes esbozado y la realidad de los centros educativos, por lo que ésta institución se encuentra en un proceso de revisión curricular para la optimización de la formación pedagógica en informática.

\section{Las prácticas profesionales en la formación docente}

El escenario para la aplicación de los conocimientos adquiridos en materia pedagógica y especializada, está ubicado en las prácticas profesionales las cuales deben ser cursadas por los estudiantes durante la carrera siguiendo una estructura que debería proporcionarle la formación necesaria para ejercer la profesión exitosamente una vez egresado de la universidad, por lo cual, deberá experimentar una serie de situaciones reales o simuladas para ir afinando la práctica y los elementos relacionados.

A este respecto, la UPEL (1984) concibe la práctica profesional como un proceso de aprendizaje y ejecución de competencias. Ese proceso es sistemático, progresivo y acumulativo en dirección a preparar al practicante para: organizar experiencias de aprendizaje, investigaciones educativas, actividades administrativas y evaluativas. Lo anterior, tiene como finalidad que el estudiante que cursa las prácticas profesionales identifique su rol como docente y las funciones inherentes a la profesión que decidió elegir.

Así pues, las experiencias que le permitirán al practicante adquirir las herramientas necesarias para desempeñarse exitosamente en las prácticas profesionales, pueden lograrse a través de tres modalidades: plan convencional, plan de experiencias no convencionales y el plan experimental (ob. cit.).

Es importante destacar que, el plan convencional es la modalidad en la que generalmente participan los estudiantes. En ella, los practicantes desarrollan la práctica en centros escolares dependientes del estado y otros institutos oficiales, así como en los de naturaleza privada.

Particularmente, en la Universidad Pedagógica Experimental Libertador (UPEL), las prácticas profesionales se estructuran en cuatro fases obligatorias: Observación, Ensayo Didáctico, Integración DocenciaAdministración y Ejecución de un Proyecto de Investigación.

En específico, la Fase de Ensayo Didáctico tiene como propósito (UPEL, 1984):

Desarrollar, corregir o consolidar
habilidades, destrezas y actitudes
orientadas hacia el proceso de
aprendizaje, mediante la ejercitación en
situaciones simuladas o reales de un
conjunto de competencias específicas y
representativas referidas a los roles de
facilitador y orientador,
fundamentalmente, pretende, además,
el fortalecimiento del compromiso
profesional, iniciado en la fase de
observación (p.5).

Aunado a ello, entre las actividades características de ésta fase se encuentra la ejecución de microclases, el autodiagnóstico del comportamiento docente y la retroalimentación. En lo concerniente a las actividades de evaluación, éstas giran en torno a la planificación de clases y microclases, la observación, actuación del practicante, informe docente, entre otras.

Por lo tanto, las prácticas profesionales constituyen un elemento neurálgico en la formación docente, donde se dispone el escenario para que el practicante ponga de manifiesto conocimientos, habilidades $\mathrm{y}$ destrezas a nivel pedagógico y especializado. 


\section{Enseñanza de la algoritmia y la programación}

La concepción de la algoritmia y programación como áreas de estudio de alta dificultad, por los niveles de abstracción que requieren, generalmente ha ocasionado predisposición en los estudiantes al cursar alguna de éstas asignaturas. A la par, el docente responsable de llevar el proceso instruccional además de poseer los conocimientos especializados y pedagógicos, también ha de implementar mecanismos para superar las barreras psicológicas de los estudiantes y acondicionar el escenario para la enseñanza de la algoritmia y programación de manera exitosa.

En concordancia, la enseñanza de cualquier área de conocimiento, particularmente la algoritmia y programación, solicita preparar a los estudiantes para el aprendizaje encaminando el proceso instruccional hacia el éxito. Cooper (2005) indica que es preciso informar a los discentes acerca de cuatro aspectos fundamentales:

- Resultado esperado.

- Actividades del estudiante.

- Actividades del maestro.

- Actividades de evaluación.

Por consiguiente, los estudiantes alcanzarán los objetivos planteados si conocen hasta donde llegarán; de allí que las actividades de enseñanza que el docente diseñe deben considerar los aspectos antes señalados. En materia de algoritmia y programación, la puesta en práctica de éstas premisas permitirá que los estudiantes sean conscientes de los esfuerzos a realizar para responder a las actividades evaluativas asociadas generalmente a la resolución de problemas.

Por su parte, Almeida, Blanco y Moreno
(2004) hacen referencia puntual a la instrucción en ésta área, al expresar que: "el aprendizaje de materias relativas a la algoritmia y la programación de algoritmos representa quizás uno de los niveles de dificultad más elevados en lo que se refiere a materias propias de la ingeniería".

La enseñanza de la programación solicita entonces comprender algunos principios importantes para encaminar a los estudiantes a un proceso instruccional atractivo $\mathrm{y}$ significativo. Entre los preceptos a considerar se encuentran (ob.cit.):

- Reconocer que la programación implica un proceso mental complejo y creativo que exige inteligencia, conocimiento, habilidades y disciplina.

- La adquisición de conocimientos se logra estudiando los conceptos, fundamentos y las técnicas básicas de programación.

Adicionalmente, López García (2007) sostiene que la destreza para solucionar problemas es un elemento primordial a desarrollar en los estudiantes en el área de algoritmia y programación, por lo que señala la existencia de estrategias generales para abordar el tema tales como: ensayo y error, iluminación, heurística, algoritmos, modelo de procesamiento de información, análisis de medios y fines, razonamiento analógico, lluvia de ideas, sistemas de producción y pensamiento lateral. De lo anterior, la resolución de problemas a través de algoritmos es la estrategia sugerida en el campo de la informática.

En síntesis, existen diferentes caminos para abordar la enseñanza de la algoritmia y programación. Puntualmente, requiere de un enfoque teórico práctico para que el estudiante avance en la adquisición de conceptos y pueda aprender técnicas específicas de programación. 


\section{Materiales instruccionales en la enseñanza de algoritmia y programación}

Los materiales instruccionales para potenciar la enseñanza de un área de conocimiento son importantes, pero más aún reconocer que el medio por sí sólo no tiene la potencia para lograr algo, lo esencial está relacionado con la integración del mismo a la práctica y su correspondencia con los contenidos curriculares (Chiarani, 2001).

Específicamente, Fernández Bravo (2005) explica que el material es un medio dirigido a generar resultados productivos en el aprendiz.

Así pues, los materiales instruccionales deben considerarse como medios que permiten suministrar información de calidad; por tal motivo deben ser cuidadosamente diseñados para atraer la atención del estudiantado y lograr que el mensaje llegue. De allí que es importante: organizar y secuenciar el contenido, considerar las características de la audiencia (clientes y usuarios) y seleccionar el formato de presentación más apropiado (Diez de Tancredi, 2001).

Por su parte, Beccaria y Rey (s.f) explican que los docentes actualmente disponen de un volumen creciente de materiales curriculares y elementos auxiliares para la enseñanza. Entre ellos se cuentan: libros, objetos concretos, mapas, películas, libros de texto, computadoras y otros.

En esta línea de ideas, Szczurek (1984) propone una taxonomía de medios que incluye: medios visuales de imagen fija y de imagen en movimiento, medios auditivos, medios audiovisuales de imagen fija y de imagen en movimiento y los medios multisensoriales.

En consecuencia, es notable la existencia de una variada gama de materiales instruccionales que deben ser seleccionados cuidadosamente para enriquecer el proceso instruccional. Particularmente, en el área de algoritmia y programación, Bruno (2005) hace referencia al gran esfuerzo que requiere la enseñanza de éstas asignaturas, por lo que es necesaria la búsqueda de recursos que permitan diversificar las estrategias de enseñanza.

Por lo tanto, queda manifiesto que el énfasis debe centrarse en el medio como apoyo al proceso instruccional más que en sus atributos y características pues establecen condiciones para que el aprendizaje se produzca. De allí que, algunos principios generales considerados por éste autor, relativos a los materiales de instrucción son:

- Cualquier tipo de medio, desde el más complejo al más elemental es simplemente un recurso didáctico, que deberá ser movilizado cuando el alcance los objetivos, los contenidos, las características de los estudiantes, en definitiva, el proceso comunicativo en el cual estemos inmersos, lo justifique.

- El aprendizaje no se encuentra en función del medio, sino fundamentalmente sobre la base de las estrategias y técnicas didácticas que apliquemos sobre él. Por ello, antes de pensar en términos de qué medio debemos plantearnos para quién, cómo lo vamos a utilizar y qué pretendemos con él.

- No existe el "supermedio". No hay medios mejores que otros, su utilidad depende de la interacción de una serie de variables y de los objetivos que se persigan, así como de las decisiones metodológicas que apliquemos sobre los mismos.

Por otra parte, la estructuración de contenidos para materiales instruccionales puede servirse de la revisión exhaustiva de diversos materiales instruccionales asociados al área en desarrollo. En relación al ámbito de 
la algoritmia y programación, López Ortiz (2005) propone el abordaje de aspectos como: introducción al hardware, software, técnicas de programación, lenguajes de programación, tipos de datos, operadores, variables, constantes, expresiones.

Igualmente, García Molina (2001) sugiere los temas básicos en el área de algoritmia y programación que incluye: las estructuras de control, iteración, arrays, ordenación, recursividad, estructuras de datos dinámicas. También señala que los estudiantes deben iniciarse con el paradigma procedural y luego pasar al paradigma orientado a objeto, pues es más natural y apropiado.

En resumen, la enseñanza de la algoritmia y programación puede encontrar en los materiales instruccionales el apoyo para potenciar la experiencia educativa de los futuros docentes en las prácticas profesionales.

\section{MÉTODO}

Este estudio está enmarcado en la modalidad de proyecto factible, apoyado en una investigación de campo. También, el estudio responde al nivel de investigación comprensivo de tipo proyectivo, donde la intencionalidad va dirigida a la generación de una alternativa de cambio en aras de dar respuesta a las necesidades detectadas (Bautista, 2006).

Igualmente se apoya en una investigación de campo, considerando que se realizó un estudio sistemático de una situación en la realidad; tal es el caso de los estudiantes de la Especialidad Informática del IPC cursantes de la Fase de Ensayo Didáctico y los profesores que administran ésta práctica profesional.

Para el desarrollo de este trabajo de investigación fue empleado el Modelo de Desarrollo Instruccional de Szczurek (1989), el cual está integrado por cuatro fases:
Estudio de Necesidades, Diseño de la Solución, Implantación del Diseño y la Evaluación de cada una de las fases.

Este desarrollo instruccional como proyecto factible, se realizó abordando sólo tres (3) de las cuatro (4) fases del modelo propuesto siendo que para la fase de Implantación se sugirió un modelo para su posterior aplicación.

\section{Procedimiento}

Se determinó la situación problemática y las causas según el contexto estudiado. Permitió obtener los datos requeridos para identificar la necesidad y avanzar hacia la proposición de la solución instruccional.

Específicamente, para la encuesta se diseñó un cuestionario como instrumento para registrar los datos. Consta de veinticinco (25) ítems con una escala de respuestas tipo Likert, la cual presenta tres (3) alternativas de respuesta: poco, medio y mucho. Además, posee una columna de especificaciones para que los encuestados pudieran complementar la respuesta de las preguntas que así lo requirieran.

El cuestionario fue aplicado a los diez (10) estudiantes de informática de Fase de Ensayo Didáctico, considerando que han cursado asignaturas asociadas a la algoritmia y programación y son quienes se enfrentan por primera vez a la posibilidad de administrar materias, en la práctica profesional, relacionadas con ésta área de conocimiento. Por tal razón, representaron una fuente de información primaria para detectar las posibles causas de la situación objeto de estudio.

La entrevista, de naturaleza semiestructurada, fue la técnica seleccionada para obtener información de los profesores que dictan las prácticas profesionales. Para tales efectos, se construyó un guion de entrevista con once (11) preguntas abiertas 
que permitieran expresar sus experiencias respecto al desempeño de los estudiantes de informática al ser asignados para dar clases de algoritmia y programación. Además, de información asociada a aspectos administrativos de las prácticas profesionales que influyen directamente en este escenario.

Los tres (3) profesores responsables de dictar las prácticas profesionales, a la Especialidad Informática del IPC, fueron entrevistados en momentos determinados siguiendo el guion de entrevista establecido.

En suma, la aplicación de los instrumentos abarco la recolección de los datos y posteriormente el análisis respectivo de los resultados arrojados por el cuestionario y la entrevista.

\section{RESULTADOS}

\section{Estudio de necesidades}

En la primera fase del Modelo de Szczurek (1989) se siguieron los pasos de las tres etapas del Modelo propuesto por Müller (2003).

\section{Detección de necesidades}

Para dar cumplimiento a la primera fase del Estudio de Necesidades se aplicaron dos instrumentos para la recolección de datos: los resultados obtenidos en la aplicación de un cuestionario a diez (10) estudiantes de la Especialidad Informática del IPC y de un guion de entrevista a tres (3) profesores que dictan la Fase de Ensayo Didáctico, permitió identificar la situación actual de las prácticas profesionales en ésta especialidad respecto a la enseñanza de asignaturas relacionadas a la algoritmia y programación.

Los resultados arrojados por el cuestionario aplicado a los estudiantes, permiten afirmar que los alumnos poseen debilidades pedagógicas en lo relativo a la elaboración de planificaciones, respecto al conocimiento y uso de técnicas, medios y recursos instruccionales; más aún cuando estos aspectos están asociados a la enseñanza de la algoritmia y programación.

También son palpables las debilidades en el área especializada, lo cual influye en su decisión de evitar la administración de asignaturas asociadas a éste campo en las prácticas profesionales.

En líneas generales, poseen información de: cómo planificar una clase, algunos han planificado, conocen algunas técnicas, medios y recursos para la enseñanza; pero cuando se trata de algoritmia y programación el vacío es considerable. Afirman conocer pero tienen poca o ninguna experiencia en la aplicación y uso de éstos tópicos.

Lo anterior, permite identificar debilidades en los estudiantes relacionadas con su preparación para dictar clases, para enseñar; más aún algoritmia y programación. Sin embargo, cabe destacar que ellos creen poseer fortalezas para comunicarse, manifiestan poseer muchas habilidades comunicativas aunque no tantas para dar clases de algoritmia y programación.

En lo respectivo a los conocimientos que poseen en ésta área de conocimiento, la generalidad de los encuestados maneja medianamente temas relacionados al área. Unos más que otros indican dominar contenidos de algoritmia y programación; en mayor grado en lo referente a la utilización de lenguajes de programación como: java, php, pascal, $\mathrm{C}$ y $\mathrm{C}++$. Aunado a ello, aunque indican poseer algún tipo de experiencia en el área no demuestran interés en mantenerse actualizados respecto al tema. Es mínima la cantidad de estudiantes que expreso consultar con frecuencia información de algoritmia y programación.

Por otra parte, en los ítems asociados a la vigencia y pertinencia de los contenidos curriculares que han cursado durante la 
carrera, la respuesta de la mayoría indica que se requiere la optimización de los contenidos curriculares y la urgencia de enfatizar en el abordaje de los contenidos durante las clases tal y como se establecen en los programas sinópticos. Ésta situación puede ser objetada, considerando que la mayoría manifestó no consultar información actualizada asociada al área; en consecuencia, vale preguntar cuáles son los criterios que toman en cuenta para señalar que los contenidos curriculares están desactualizados.

A la par, algunos estudiantes sugieren que les puede resultar sencillo los contenidos de algoritmia y programación pero su enseñanza les puede resultar menos fácil. Específicamente, para enseñar algoritmia y programación señalan que les hace falta fortalecer las debilidades en el área de conocimiento, superar miedos asociados al tema $y$ hacerse de herramientas y conocimientos para enseñar éstos contenidos.

Además, los resultados revelaron que son pocos a quienes les motiva enseñar algoritmia y programación en las prácticas profesionales de la Fase de Ensayo Didáctico, tomando en cuenta las debilidades para administrar cursos de ésta área, entre ellas: falta de estrategias para enseñar el tema, desconocimiento profundo de algunos contenidos, falta de computadoras y recursos en los centros de aplicación, falta de preparación, adecuación de estrategias para enseñar el área, falta de experiencia docente.

En suma, es palpable la necesidad instruccional asociada a vigorizar la preparación pedagógica y especializada de los estudiantes para asumir cursos de algoritmia y programación en las prácticas profesionales. En específico, en el qué y cómo enseñar ésta área neurálgica de la informática.

Adicionalmente, los encuestados dejaron identificar debilidades en el manejo de los contenidos propios de la algoritmia y programación; lo cual también representa una de las razones por las que ésta área de estudio no ocupa el primer lugar de opciones para dar clases en la Fase de Ensayo Didáctico.

Así pues, por la naturaleza de éste estudio la solución instruccional a la necesidad detectada debe ir en aras de ofrecer a los estudiantes alternativas para que puedan asumir la administración de asignaturas en el área en cuestión saldando las carencias que puedan tener a nivel pedagógico $\mathrm{y} / \mathrm{o}$ especializado. En otras palabras, es necesario ofrecerles alternativas dirigidas a minimizar las debilidades pedagógicas y especializadas que poseen.

La información suministrada, siguiendo un guion de entrevista, por tres (3) de los profesores que administran la Fase de Ensayo Didáctico, permitió conocer sus opiniones acerca de la administración de asignaturas asociadas a la algoritmia y programación, por parte de los estudiantes de la Especialidad Informática del IPC, en las prácticas profesionales de ésta segunda fase que deben cursar los alumnos.

Tomando en consideración que, la información suministrada por los profesores de la Fase de Ensayo Didáctico coincide altamente; es deducible que la administración de cursos asociados a la algoritmia y programación tienen que ver con aspectos pedagógicos y especializados relativos a las debilidades y/o fortalezas que presentan los estudiantes cuando cursan las prácticas profesionales.

Las carencias pedagógicas y/o especializadas que muestran los estudiantes de sexto semestre, en teoría, cuando van a cursar Fase de Ensayo Didáctico, están directamente relacionadas a que no saben planificar, tienen deficiencias en diferenciar técnicas, medios y recursos instruccionales, presentan dificultades para administrar 
tiempo en las planificaciones, poseen debilidades en el área de algoritmia y programación inclinándose por aspectos teóricos más que prácticos y finalmente manifiestan indisposición a asumir cursos relacionados con ésta área de conocimiento por insuficiencia de conocimientos en el área. La tendencia general es a preferir dar clases de ofimática, Internet, páginas Web, HTML, arquitectura del computador y aplicaciones de código abierto; sólo en casos particulares y escasos hay quienes se inclinan a dar programación o algoritmia.

Los profesores de Fase de Ensayo Didáctico, determinan el nivel de conocimiento que poseen los estudiantes, antes de ser asignados a los centros de aplicación, mediante las microclases que los alumnos realizan abordando determinados contenidos de informática. Por lo tanto, tomando en cuenta las opiniones expresadas por los profesores de la fase, las razones de la casi nula tendencia de los practicantes a dar ésta área de conocimiento se resumen en la falta de conocimientos en el área y la falta de competencias pedagógicas.

Como información complementaria, los entrevistados señalan la existencia de un desfase curricular donde los estudiantes cursan en paralelo a la fase la asignatura estrategias y recursos instruccionales y no han visto evaluación, además de que ya aprobaron planificación pero demuestran grandes debilidades al respecto. Éste aspecto curricular, antes esbozado, sirve de referencia para comprobar la formación que han recibido los estudiantes para enfrentarse a sus primeras experiencias docentes en las prácticas profesionales de Fase de Ensayo Didáctico.

Adicionalmente, entre los aspectos administrativos relacionados con la asignación de practicantes para dar clases de informática y específicamente de algoritmia y programación se encuentran: centros de aplicación donde impartan la Especialidad Informática, centros de aplicación que acepten practicantes del Instituto Pedagógico de Caracas, centros de aplicación que tengan horarios compatibles con la disponibilidad del estudiante, centros de aplicación de fácil acceso para los practicantes, centros de aplicación que den prioridad a los practicantes del IPC y no de otras universidades, entre otras eventualidades que saldar a nivel administrativo.

En tal sentido, al contrastar los resultados obtenidos en la aplicación del cuestionario a los estudiantes que cursaron Fase de Ensayo Didáctico y la información suministrada por los profesores entrevistados que administran esta fase, es posible afirmar que existen necesidades instruccionales que requieren soluciones a la brevedad posible.

Por una parte, los estudiantes manifiestan tener poca motivación a dar clases de algoritmia y programación por las debilidades que posean en el área; y por otro lado la confirmación de los docentes que administran la Fase de Ensayo Didáctico de una problemática asociada a la administración de cursos de éstas áreas en las prácticas profesionales de ésta fase.

En síntesis, los aspectos comunes y complementarios citados por profesores de ésta práctica profesional, en contraste con las opiniones de los estudiantes, permite afirmar la existencia de la necesidad instruccional de optimizar la preparación pedagógica y especializada de los practicantes para dar clases de algoritmia y programación.

\section{Proposición de soluciones factibles}

La alternativa de solución que se consideró más adecuada, para dar respuesta a la necesidad instruccional detectada, fue la del diseño de un material instruccional para la enseñanza de la algoritmia y programación 
que permita al estudiante de informática, cursante de la Fase de Ensayo Didáctico, optimizar la preparación y ejecución de las clases asociadas a esta área de conocimiento siguiendo las orientaciones pedagógicas y especializadas descritas en el mismo.

Dicha solución es factible ya que, existe disposición de los profesores de las prácticas profesionales a sugerir su uso, fue diseñada una propuesta de presentación del material a los estudiantes para explicar su utilización, su diseño permite su uso fácil y flexible, perdura en el tiempo y es económicamente accesible pues puede ser reproducido por los estudiantes con fines académicos.

El diseño del material instruccional se realizó bajo el modelo propuesto por Dorrego (1994) descrito en apartados anteriores. También, se consideró pertinente la planificación de una clase para presentar el material instruccional a los estudiantes y orientar su uso. Para lo anterior, se utilizó el Modelo de Aprendizaje a Través de la Práctica propuesto por Shank, Berman y Mcpherson (citado por Reigeluth, 1999). Es importante destacar que, la clase propuesta para presentar el material instruccional es una iniciativa de la autora de ésta investigación considerando la dinámica de la asignatura Fase de Ensayo Didáctico.

Así pues, la solución instruccional presenta un gran alcance debido a que abarca aspectos pedagógicos y especializados para la enseñanza de la algoritmia y programación en las prácticas profesionales de la Fase de Ensayo Didáctico, todo en aras de obtener resultados positivos que puedan perdurar en el tiempo.

\section{Diseño de la solución (material instruccional)}

El material instruccional son medios que buscan generar resultados positivos en quien aprende (Fernández Bravo, 2005). Según la naturaleza de ésta investigación, se consideró el diseño de un material instruccional en formato impreso así como la planificación de una clase para presentar el mismo; lo anterior, como parte de la solución instruccional para encaminar a los estudiantes en la enseñanza de la algoritmia y programación en las prácticas profesionales de la Fase de Ensayo Didáctico.

Como parte de la asignatura y según la dinámica del curso, se pretende potenciar el trabajo de los practicantes de informática. Así pues, se considera que mediante el uso del material instruccional, es posible atender las necesidades instruccionales de los estudiantes, de tal manera de brindar la información necesaria y con los recursos para aumentar el interés y que el estudiante reflexione sobre lo que hace y ha aprendido.

La estructura general del diseño de instrucción de la solución será descrita detalladamente en el próximo capítulo.

\section{Implantación del diseño de la solución}

En concordancia con la tercera fase del Modelo de Desarrollo Instruccional de Szczurek (1999) referida a la implantación, contempla la ejecución y puesta en práctica de la solución diseñada con el objetivo de satisfacer la necesidad detectada. Lo anterior, requiere de un plan que garantice el logro del diseño instruccional, para lo cual se propone el modelo de Zabatta (1997).

De allí que, para diseñar el plan de implantación siguiendo el modelo señalado, es importante destacar que la propuesta en factible técnica, operativa y económicamente. En primera instancia porque se dispone de los medios para su construcción, existen practicantes y profesores que cada semestre abordan la enseñanza de la algoritmia y programación, adicionalmente la construcción y reproducción del material es económicamente accesible. 
Los aspectos antes descritos, servirán de insumo para la elaboración futura de un plan de implantación de la solución instruccional propuesta; tomando en cuenta que en ésta investigación solo abarca el diseño de la solución.

\section{Evaluación}

Estudio de necesidades: Según el Modelo de Evaluación de Sttuflebeam (1987, citado por Abdala, 2007), la primera fase corresponde a la evaluación de las necesidades asociadas a la situación actual de los estudiantes con respecto a objetivos previamente planteados y la discrepancia entre lo deseable y la realidad. La evaluación del contexto permitió describir el ambiente donde se encuentra inmerso el sistema en estudio.

En consecuencia, la evaluación del Estudio de Necesidades se llevó a cabo con la triangulación de la información obtenida de los instrumentos aplicados, como el cuestionario y el guion de entrevistas y la revisión de las referencias documentales y electrónicas. Dio como resultado la solución instruccional.

Diseño: La identificación de insumos que permitieran el diseño de la solución instruccional, fue posible luego de detectada la necesidad. Los resultados arrojados por los instrumentos aplicados permitió la selección de una solución instruccional factible cónsona con el sistema en estudio.

En correspondencia con el modelo de evaluación, las decisiones de esta fase son de planeación. En específico, fueron considerados los recursos disponibles y el procedimiento para el diseño del material instruccional y la planificación de una clase para la presentación del mismo ante los practicantes que harán uso de él.

Evaluación: La evaluación del producto, denominación de esta fase según el Modelo de
Evaluación de Sttuflebeam (1987, citado por Abdala, s.f), abarca desde el estudio de necesidades hasta la implantación de la solución. A efectos de ésta investigación, la fase de implantación no se realizó en consecuencia no procede la evaluación de ésta.

En este aparte se asocian: resultados obtenidos una vez transformados los insumos (diseño del material instruccional), relación con objetivos esperados y expectativas del contexto.

En síntesis, la evaluación del todo el proceso incluyendo la participación de juicio de expertos en la validación de los instrumentos para la detección de necesidades y de la validación del diseño del material instruccional, las sugerencias de los profesores que administran la Fase de Ensayo Didáctico y las decisiones tomadas en el desarrollo de la investigación; permitieron la retroalimentación constante y el ajuste de los aspectos necesarios para el avance óptimo hacia la solución instruccional planteada.

\section{CONCLUSIONES}

Las conclusiones derivadas de ésta investigación se presentan a continuación, siguiendo las fases del Modelo de Desarrollo Instruccional de Sczurek (1989) cubiertas en éste estudio:

En las prácticas profesionales, ejecutadas por los estudiantes de la Especialidad Informática del IPC, de la Fase de Ensayo Didáctico, la mayoría de los alumnos manifiesta poseer debilidades pedagógicas y especializadas para asumir la administración de asignaturas asociadas a la algoritmia y programación en sus primeras experiencias docentes durante la fase. Es de hacer notar que el análisis permitió detectar contradicciones manifestadas donde manifestaban que sabían y luego no. En la última interrogante se pudo constatar tales resultados. 
De igual manera, los profesores responsables de administrar la Fase de Ensayo Didáctico confirmaron tal realidad. Las debilidades de los estudiantes a nivel pedagógico abarcan la planificación, técnicas y recursos instruccionales. En el ámbito especializado tienen nociones de algoritmia y programación aunque ya han cursado tres materias en este sentido, se inclinan por la teoría y demuestran debilidades en esta área de conocimiento. Debido a las deficiencias pedagógicas y especializadas los estudiantes evitan dar clases de algoritmia y programación.

Se consideró el diseño de un material instruccional en formato impreso, ya que contribuirá a minimizar las debilidades detectadas en aras de mejorar sus primeras experiencias docentes. Los instrumentos aplicados, la revisión de diferentes fuentes y la evaluación del sistema en estudio proporcionaron información valida que sustento el diseño de la alternativa de solución seleccionada.

El cumplimiento de las fases del Modelo de Desarrollo Instruccional seleccionado favoreció altamente dicha solución.

Proporcionó información con respecto a las necesidades de los estudiantes en cuanto a su formación académica para dar clases de algoritmia y programación.

Permitió evidenciar la factibilidad de la solución, a través de la consecución de los objetivos propuestos. Por consiguiente, se logró elaborar una propuesta direccionada a ofrecer orientaciones, un material de apoyo de referencia para ubicar al estudiante y darle herramientas para avanzar. La innovación permanente del docente en las instituciones educativas garantiza la construcción e incorporación en nuevos paradigmas. Incremento de la disposición para superar exigencias y limitaciones en la formación de profesionales comprometidos con el cambio.

\section{REFERENCIAS}

Abdala, E. (2007). La evaluación de impacto: tipos, modelos teóricos y proceso técnico. [Documento en Línea]. Disponible: http://www.educarchile.cl/Userfiles/P00 01/File/La\%20evaluaci\%C3\%B3n\%20de \%20impacto.pdf [Consulta: 2018, octubre 5]

Almeida, F., Blanco, V. y Moreno, L.M. (2004). EDAApplest: Una herramienta Web para la Enseñanza de Estructura de Datos y Técnicas Algorítmicas [Documento en línea]. Disponible: http://bioinfo.uib.es/ joemiro/aenui/pr ocJenui/Jen2004/ponencias/ponencia48. pdf [Consulta 2018, junio 10]

Bautista, E. (2006). Manual de Metodología de Investigación. Caracas: Talitip

Beccaria, L. P. y Rey, P. E. (s.f). La Inserción de la Informática en la Educación y sus Efectos en la Reconversión Laboral. [Documento en línea]. Disponible: http://www.c5.cl/ieinvestiga/actas/ribie 96/Colombia.html [Consulta 2019, junio 10]

Bruno, O. R. (2005). Análisis de la percepción de los alumnos y de los docentes para la incorporación de un sistema tutor inteligente como facilitador del aprendizaje de algoritmia. [Documento en línea]. Disponible: http://laboratorios.fi.uba.ar/lie/Revista/ Articulos/020204/A1mar2005.pdf [Consulta 2019, junio 10]

Chiarani, M. C. (2001). El Medio Informático desde la escuela como unidad de cambio. Revista Iberoamericana de Educación [Revista en Línea]. Disponible: http://www.rieoei.org/deloslectores/17 6Chiarani.PDF [Consulta: 2018, octubre 5]

Cooper, J. (2005) Estrategias de Enseñanza: Guía para una mejor instrucción. México: Limusa Noriega Editores

Diez de Tancredi, D. (2001). Una propuesta metodológica para la producción de medios educativos. Revista de Investigación Vol. 48, 11.

Dorrego, E. (1994). Modelo para la producción y evaluación formativa de medios instruccionales, aplicado al vídeo y al software. [Documento en Línea]. Disponible: 
http://www.c5.cl/ieinvestiga/actas/ribie 94/II_72_84.html [Consulta: 2019, junio 5]

Fernández Bravo, J. A. (2005). Avatares y Estereotipos sobre la enseñanza de los algoritmos en matemáticas. Revista Iberoamericana de Educación Matemática [Revista en Línea]. Disponible: http://www.fisem.org/web/union/revist as/4/Union_004_006.pdf [Consulta: 2019, junio 5]

García Molina, J. J. (2001). Avatares y Estereotipos sobre la enseñanza de los algoritmos en matemáticas. [Documento en Línea]. Disponible: http://bioinfo.uib.es/ joemiro/aenui/pr ocJenui/ProcWeb/actas2001/gaesc43.pd f [Consulta: 2019, junio 5]

López, García, J. C. (2007). Algoritmos y Programación para Docentes. [Documento en Línea]. Disponible: http://www.eduteka.org/pdfdir/Algorit mosProgramacion.pdf [Consulta: 2018, junio 5]

López Ortiz, I. A. (2005). Curso de Algoritmos. [Documento en Línea]. Disponible:http://www.monografias.com /trabajos-pdf4/algoritmoscurso/algoritmos-curso.pdf [Consulta: 2019, junio 5]

Müller, G. (2003). Estudio de Necesidades: Una Metodología para Introducir Cambios. Tópica Extensa. Año 3. N-1

Oviedo, M. (2002). Optimización Dinámica en Tiempo Discreto. Ecuación de Bellman [En línea] p. 12

Pereira, M. (2005). Condicionamientos sociales que determinan la existencia de la Asignatura Base de Datos en la Formación de los Profesores de Informática. Revista Científico Pedagógica [Revista en línea]. Disponible: file://C:/Users/USUARIO/Downloads/D ialnet-

CondicionamientosSocialesQueDetermina nLaExistencia-6320437.pdf [Consulta 2019, septiembre 10]
Punceles, L. I. (2008, Mayo). Modelo de Formación Pedagógica Continuo dirigido a los docentes de educación superior que respondan a los nuevos retos de la educación superior contemporánea. Ponencia presentada en el Encuentro Kipus de Formación Docente, Barquisimeto.

Reigeluth, C. M. (1999). What is instructionaldesign theory and how is it changing. Instructional-design theories and models: A new paradigm of instructional theory, $2,5-29$

Szczurek, M. (1999). Simulaciones y juegos Instruccionales. Un hipertexto. Trabajo de ascenso. Universidad Pedagógica Experimental Libertador. Instituto Pedagógico de Caracas. Trabajo inédito

Szczurek, M. (1984). La Selección de Medios en el Diseño Instruccional. Revista de Investigación Educacional, 8(18), 85-93, 1984

Szczurek, M. (1989). La estrategia instruccional. Revista de Investigación y Postgrado, 4(2)

Universidad Pedagógica Experimental Libertador. (1984). InstructivoNormativo para la realización $y$ evaluación de la práctica profesional. Caracas: Autor.

Universidad Pedagógica Experimental Libertador, Vicerrectorado de Docencia. (2002). Diseño Curricular Especialidad: Informática. Caracas: Autor.

Universidad Pedagógica Experimental Libertador. (2006) Manual de Trabajos de Grado, de Especialización y Maestrías y Tesis Doctorales. 4⿳亠丷a ed. Caracas: Fedeupel.

Zabatta, V. (1997). Implantación Efectiva. Mimeografiado. Caracas. Universidad Pedagógica Experimental Libertador. Instituto Pedagógico de Caracas 